\title{
Modélisation et optimisation de la photodégradation du 4-méthylphénol dans un réacteur à recirculation en présence d'UV/ZnO
}

\section{Modelisation and optimisation of the photodegradation of 4-methylphenol in a recirculating batch reactor using $\mathrm{UV} / \mathrm{ZnO}$}

\author{
Smail Merabet, Abdelkrim Bouzaza, Mohamed Bouhelassa et Dominique \\ Wolbert
}

Volume 22, numéro 4, 2009

URI : https://id.erudit.org/iderudit/038331ar

DOI : https://doi.org/10.7202/038331ar

Aller au sommaire du numéro

Éditeur(s)

Université du Québec - INRS-Eau, Terre et Environnement (INRS-ETE)

ISSN

1718-8598 (numérique)

Découvrir la revue

Citer cet article

Merabet, S., Bouzaza, A., Bouhelassa, M. \& Wolbert, D. (2009). Modélisation et optimisation de la photodégradation du 4-méthylphénol dans un réacteur à recirculation en présence d'UV/ZnO. Revue des sciences de l'eau / Journal of Water Science, 22(4), 565-573. https://doi.org/10.7202/038331ar
Résumé de l'article

L'étude de la photodégradation du 4-méthylphénol a été menée sur un pilote à recirculation. Cette molécule a été prise comme composé modèle pour le traitement des effluents de l'industrie avicole. Ce travail a consisté en l'optimisation et la modélisation de l'élimination du 4-méthylphénol par photocatalyse en présence de ZnO. L'utilisation des plans d'expériences, et en particulier de la méthodologie de surface de réponse (RSM) et un plan central composite (CCD), a permis la détermination de l'influence des effets simultanés et de l'interaction des paramètres opératoires sur le rendement de la photodégradation. Les paramètres étudiés sont la concentration initiale en 4-méthylphénol, la concentration en catalyseur et le débit de recirculation de la solution.

Les résultats montrent que l'application de la RSM permet de décrire d'une manière correcte l'influence de ces trois paramètres expérimentaux sur l'efficacité du traitement. Les valeurs optimales des paramètres donnant un rendement maximal (100 \%) ont pu être déterminées. Les modèles de second ordre obtenus, pour le rendement de dégradation et pour l'abattement de DCO, ont été validés en utilisant différentes approches statistiques. L'utilisation de la méthode ANOVA a montré que les modèles sont hautement significatifs et en bonne adéquation avec les résultats expérimentaux. 


\title{
MODÉLISATION ET OPTIMISATION DE LA PHOTODÉGRADATION DU 4-MÉTHYLPHÉNOL DANS UN RÉACTEUR À RECIRCULATION EN PRÉSENCE D’UVIZNO
}

\begin{abstract}
Modelisation and optimisation of the photodegradation of 4-methylphenol in a recirculating batch reactor using $\mathrm{UV} / \mathrm{ZnO}$
\end{abstract}

\begin{abstract}
SMail Merabet ${ }^{1}$, AbDelKrim Bouzaz 2 $^{2 *}$, Mohamed Bouhelass ${ }^{3}$, Dominique Wolbert ${ }^{2}$
${ }^{1}$ Laboratoire d’hydraulique appliquée et environnement, Université A. Mira, 06000 Bejaïa, Algérie

${ }^{2}$ Laboratoire « Chimie et Ingénierie des Procédés », UMR 6226 « sciences chimiques de Rennes », École Nationale Supérieure

de Chimie de Rennes, 35700 Rennes, France

${ }^{3}$ Département de chimie industrielle, Université Mentouri, 25000 Constantine, Algérie
\end{abstract}

Reçu le 31 janvier 2008, accepté le 2 mars 2009

\section{RÉSUMÉ :}

L'étude de la photodégradation du 4-méthylphénol a été menée sur un pilote à recirculation. Cette molécule a été prise comme composé modèle pour le traitement des effluents de l'industrie avicole. Ce travail a consisté en l'optimisation et la modélisation de l'élimination du 4-méthylphénol par photocatalyse en présence de $\mathrm{ZnO}$. L'utilisation des plans d'expériences, et en particulier de la méthodologie de surface de réponse (RSM) et un plan central composite (CCD), a permis la détermination de l'influence des effets simultanés et de l'interaction des paramètres opératoires sur le rendement de la photodégradation. Les paramètres étudiés sont la concentration initiale en 4-méthylphénol, la concentration en catalyseur et le débit de recirculation de la solution.

Les résultats montrent que l'application de la RSM permet de décrire d'une manière correcte l'influence de ces trois paramètres expérimentaux sur l'efficacité du traitement. Les valeurs optimales des paramètres donnant un rendement maximal (100\%) ont pu être déterminées. Les modèles de second ordre obtenus, pour le rendement de dégradation et pour l'abattement de DCO, ont été validés en utilisant différentes approches statistiques. L'utilisation de la méthode ANOVA a montré que les modèles sont hautement significatifs et en bonne adéquation avec les résultats expérimentaux.

Mots clés : photocatalyse, 4-méthylphénol, RSM, CCD, optimisation.

\begin{abstract}
The study of the photodegradation of 4-methylphenol was carried out in a recirculating pilot plant. This compound is present in the liquid processing waste of the poultry industry. This work consisted of the optimization and the modelling of the elimination of 4-methylphenol by photocatalysis in the presence of $\mathrm{ZnO}$. The use of a design of experiments (DOE)
\end{abstract}

*Auteur pour correspondance:

Téléphone: $\quad 33.2 .23 .23 .80 .56$

Télécopie : $\quad 33.2 .23 .23 .81 .20$

Courriel : Abdelkrim.Bouzaza@ensc-rennes.fr 
approach, and in particular the response surface methodology (RSM) and a central composite design (CCD), allowed the determination of the influence of the simultaneous effects and the interaction of the operational parameters on the degree of photodegradation. The studied parameters were the initial concentration of 4-methylphenol, the catalyst concentration and the solution recirculation flow. The RSM application made it possible to describe the influence of these three experimental parameters on the effectiveness of the treatment. The optimal parameter values leading to maximum efficiency (100\%) were determined. The second-order models obtained for the degradation output and the reduction of COD were validated using various statistical approaches. The use of an ANOVA method showed that the models were highly significant and in good agreement with the experimental results.

Keywords: photocatalysis, 4-methylphenol, RSM, CCD, optimization.

\section{INTRODUCTION}

Les effluents de l'industrie agroalimentaire sont une des causes principales de la pollution et de la contamination des eaux de surface et souterraines. Leur traitement, avant rejet, par les méthodes conventionnelles ne permet pas la minéralisation totale des polluants contenus dans l'effluent. Leur rejet dans la nature n'est pas sans conséquence sur l'environnement. De nouvelles technologies sont nécessaires pour éliminer et décomposer complètement de tels polluants organiques et minimiser leurs effets sur la nature. La technologie la plus attrayante et la plus prometteuse semble être la photocatalyse. L'utilisation de la photocatalyse hétérogène pour l'oxydation des polluants organiques et inorganiques dans l'eau et l'air a été intensément étudiée durant ces vingt dernières années (OLLIS et AL-EKABI, 1993; SCHIAVELLO, 1997). Ce procédé, faisant partie de la famille des procédés d'oxydation avancée (POA), utilise des semi-conducteurs, tels que $\mathrm{TiO}_{2}$ et $\mathrm{ZnO}$, et peut mener à la minéralisation totale des composés organiques en $\mathrm{CO}_{2}$, eau et acides minéraux.

Bien que le $\mathrm{TiO}_{2}$ soit le photocatalyseur le plus utilisé pour la dégradation d'un large éventail de composés organiques, le $\mathrm{ZnO}$ peut représenter une alternative intéressante. En effet, d'après PIRKANNIEMI et SILLANPAA (2002), le mécanisme de photodégradation est semblable à celui du $\mathrm{TiO}_{2}$. De plus, le $\mathrm{ZnO}$ peut s'avérer plus efficace pour la dégradation de certains composés organiques tels que le 2-phénylphénol (KHODJA et al., 2001) et le phénol (MARCI et al., 2001). Cependant, certaines études signalent que le $\mathrm{ZnO}$ pourrait perdre de son efficacité en raison d'un phénomène de photocorrosion (YASSITEPE et al., 2008). Cependant, ceci ne semble pas rédhibitoire pour des utilisations de courtes durées. Le $\mathrm{ZnO}$ retrouve ses capacités initiales après un court séjour dans une solution aqueuse isolée du rayonnement lumineux.

L'objectif de cette étude consiste à étudier la photodégradation du 4-méthylphénol en présence d'UV/ $\mathrm{ZnO}$. L'influence de la concentration en polluant, de la quantité de catalyseur et du débit de circulation de la solution sur les performances du procédé sera appréhendée. En utilisant la méthodologie des surfaces de réponse (RSM) et un plan central composite (CCD) (MONTGOMERY, 2001), une modélisation des effets de ces différents paramètres sur le rendement d'élimination et sur l'abattement de la demande chimique en oxygène (DCO) sera réalisée. Cette méthodologie, appelée aussi analyse multivariable, a été employée dans la conception expérimentale de la réaction de Fenton (HERRERA et al., 2000; OLIVEROS et al., 1997) et dans le traitement photocatalytique des effluents industriels (PEREZ et al., 2001) (KUMAR et KANMANI, 2006; CHO et ZOH, 2007).

Notre démarche pourra être résumée en trois étapes essentielles. Une première, durant laquelle le nombre et les niveaux des paramètres à tester sont choisis. Un modèle polynomiale sera proposé et sa validité discutée. Une deuxième étape, basée sur l'utilisation des graphes des effets des facteurs, permettra d'évaluer les effets des différents paramètres sur les performances du procédé. Enfin, en dernière étape, une optimisation et une identification des interactions seront réalisées grâce aux graphes de contours.

En s'appuyant sur cette méthode, on peut faire ressortir l'effet de la concentration initiale en 4-méthylphénol, de la teneur en catalyseur et du débit de circulation de la solution sur les rendements de dégradation et sur l'abattement en DCO. On peut également, déterminer les interactions des paramètres étudiés et les conditions optimales de fonctionnement du procédé. Grâce à cette approche, le nombre d'essais nécessaires est réduit au minimum.

\section{MATÉRIELS ET MÉTHODE}

\subsection{Matériel}

Le photoréacteur utilisé se présente sous la forme d'un cylindre à double parois en verre (Figure 1). Le volume utile du réacteur est de $1200 \mathrm{~mL}$. La source d'irradiation est une lampe UV de marque Phillips référencée PL-L 24W/10/4P. Son spectre d'émission est compris entre 350 et $390 \mathrm{~nm}$ avec un maximum à $365 \mathrm{~nm}$. Elle est placée au centre du photoréacteur. La circulation de la solution est assurée par une pompe reliée à un débitmètre qui permet d'ajuster le débit. Ce dernier varie de $0,5 \mathrm{~mL} \cdot \mathrm{s}^{-1}$ à $4,03 \mathrm{~mL} \cdot \mathrm{s}^{-1}$. 
(1)Réacteur

(2) Pompe à eau

(3)Réservoir

(4)Lampe UV

(5)Débitmètre

(6) Vanne

(7)Plaque d'agitation

(8)Pompe à air

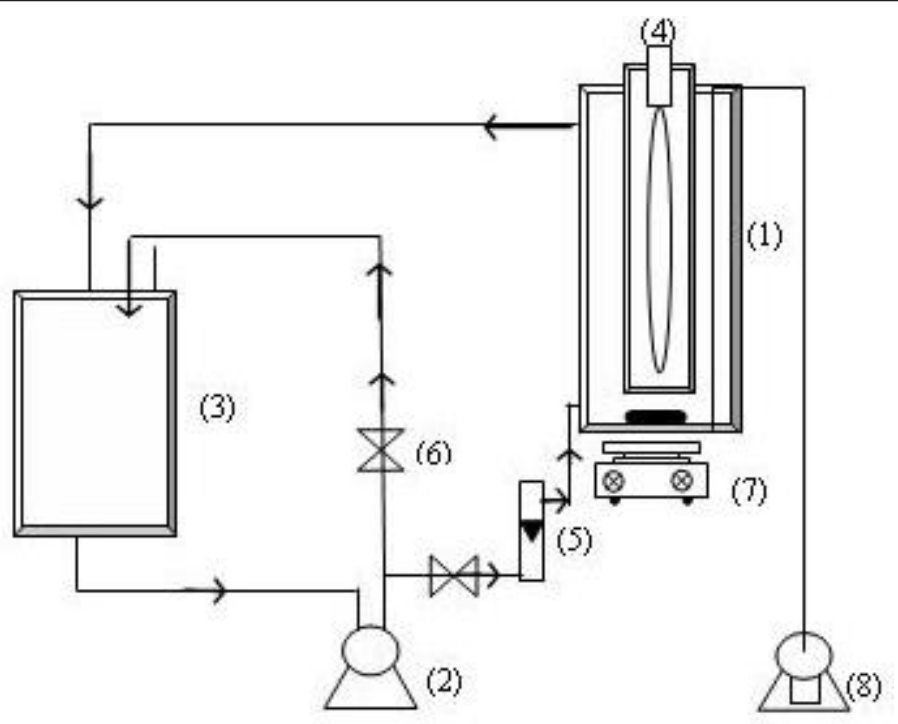

Figure 1 : Schéma du dispositif expérimental. Experimental design.

Le 4-méthylphénol est fourni par la société FLUKA, il a une pureté de $99 \%$. Appelé aussi para-crésol ou p-crésol, sa formule chimique est $\mathrm{CH}_{3} \mathrm{C}_{6} \mathrm{H}_{4} \mathrm{OH}$. Le catalyseur utilisé est l'oxyde de zinc $(\mathrm{ZnO})$ en poudre de la société Merck. Les concentrations en 4-méthylphénol testées varient de 30 à $150 \mu \mathrm{L} \mathrm{L}^{-1}$ d'eau bidistillée. La concentration de $\mathrm{ZnO}$ dans le photoréacteur varie de 0,5 à $3 \mathrm{~g} \bullet \mathrm{L}^{-1}$.

Le mélange 4-méthylphénol/ZnO est agité pendant 60 minutes à l'obscurité de façon à atteindre l'équilibre d'adsorption. La lampe UV est ensuite allumée $(t=0)$. Simultanément, on fait barboter un débit d'air de $2,5 \mathrm{~L} \cdot \mathrm{min}^{-1}$ dans la solution de façon à assurer un apport régulier d'oxygène. Les expériences ont été effectuées à $\mathrm{pH}=6,8$ et à une température de $25^{\circ} \mathrm{C}$. Le $\mathrm{pH}$ et la température sont contrôlés avec un appareil multi-paramètre Inolab.

Le suivi de la concentration en polluant est réalisé par prélèvements réguliers d'échantillons de $0,5 \mathrm{~mL}$ qui sont centrifugés, filtrés (Millipore $0,45 \mu \mathrm{m}$ ) et analysés à l'aide d'un spectrophotomètre UV-Vis Shimadzu. La longueur d'onde d'absorption maximale est de $277 \mathrm{~nm}$. L'estimation de la DCO a aussi été évaluée par la méthode standard (AMERICAN PUBLIC HEALTH ASSOCIATION, 1998).

Le rendement d'élimination du polluant est ainsi déduit par l'expression suivante : $\frac{Y_{i}-Y_{120}}{Y_{i}}$ où $Y_{i}$ désigne la concentration initiale et $Y_{120}$ la concentration atteinte au bout de 120 minutes de traitement. Le même type d'expression est utilisé dans le cas du calcul de l'élimination de la DCO.

\subsection{Méthodologie des plans d'expérience}

La méthodologie classiquement utilisée pour étudier l'influence de paramètres opératoires sur une variable d'intérêt consiste à modifier la valeur d'un paramètre tout en maintenant les autres fixes. L'exploitation des résultats et l'étude expérimentale peuvent être fortement simplifiées en utilisant la méthodologie des plans d'expérience. Cette technique permet de créer un modèle statistiquement significatif d'un phénomène qui intègre les interactions entre les variables tout en optimisant le nombre d'essais.

La méthodologie des surfaces de réponse (RSM) est une technique statistique empirique utilisée pour l'analyse de régression multiple des données quantitatives obtenues à partir des expériences statistiquement conçues en résolvant les équations multivariables simultanément. La représentation graphique de ces équations s'appelle surfaces de réponse, et permet de décrire l'effet individuel et cumulatif des variables d'essai sur la réponse et de déterminer l'interaction mutuelle entre les variables d'essai et leur effet sur la réponse (KHURI et CORNEL, 1987). L'objectif principal de la RSM est de déterminer les conditions opérationnelles optimales pour un système donné qui satisfasse les conditions spécifiques opératoires (MYERS et MONTGOMERY, 2002).

Le concept de surface de réponse modélise une variable dépendante $Y$, dite variable de réponse, en fonction d'un certain nombre de variables indépendantes (facteurs), $\mathrm{x}_{1}, \mathrm{x}_{2}$, $\ldots, \mathrm{x}_{\mathrm{k}}$, permettant d'analyser l'influence et l'interaction de ces 
dernières sur la réponse. On peut ainsi écrire le modèle pour une réponse donnée $(\mathrm{Y})$ sous la forme suivante :

$$
Y=\beta_{0}+\sum_{i=1}^{k} \beta_{i} \cdot x_{i}+\sum_{i=1}^{k} \beta_{i i .} \cdot x_{i}^{2}+\sum_{i=1}^{k-1} \sum_{j=i+1}^{k} \beta_{i j} x_{i} x j+\varepsilon
$$

où $\mathrm{Y}$ est la réponse observée, $\beta_{0}, \beta_{\mathrm{i}}, \beta_{\mathrm{ij}}, \beta_{\mathrm{ii}}$ représentent respectivement le terme constant, les coefficients des termes linéaires, des termes représentant les interactions entre variables et des termes quadratiques, et $\varepsilon$ représente l'erreur résiduelle.

La méthode des moindres carrés permet de déterminer l'estimateur de $\beta$, une matrice qui minimise la somme des carrés des erreurs résiduelles et ses coefficients sont donnés par l'équation (2).

$$
\hat{\beta}=\left(x^{t} x\right)^{-1} x^{t} Y
$$

où $\mathrm{x}$ est la matrice d'expérience, $\mathrm{x}^{\mathrm{t}}$ la transposée de $\mathrm{x}$ et $\left(\mathrm{x}^{\mathrm{t}} \mathrm{x}\right)^{-1}$ est l'inverse de la matrice $\left(\mathrm{x}^{\mathrm{t}} \mathrm{x}\right)$;

Y est le vecteur des réponses observées, représentant le pourcentage de photodégradation, utilisant les variables indépendantes $\mathrm{x}_{1}$ (Concentration en para-crésol), $\mathrm{x}_{2}$ (Charge en catalyseur) et $\mathrm{x}_{3}$ (Débit de circulation) en valeurs codées.

Le modèle de prédiction est alors donné par l'équation (3) suivante :

$$
\mathrm{Y}_{\text {pred }}=\hat{\beta}_{0}+\sum_{\mathrm{i}=1}^{3} \hat{\beta}_{\mathrm{i} . \mathrm{Xi}}+\sum_{\mathrm{i}=1}^{3} \uparrow \hat{\beta}_{\mathrm{ii} . \mathrm{x}_{\mathrm{i}}^{2}}^{2}+\sum_{\mathrm{i}=1}^{2} \sum_{\mathrm{j}=\mathrm{i}+1}^{3} \hat{\beta}_{\mathrm{ij}} \mathrm{xi} \mathrm{xj}
$$

Afin de tester la validité du modèle, l'analyse de variance (ANOVA) est utilisée afin d'examiner la signification et l'adéquation du modèle. Ce dernier permet de tracer les surfaces de réponse, d'estimer l'influence et l'interaction simultanées des facteurs sur le rendement photocatalytique.

Comme les facteurs sont en général exprimés dans des unités différentes, leurs effets ne sont comparables que s'ils sont codés. Les valeurs des variables codées sont définies de la manière suivante :

$$
\mathrm{x}_{\mathrm{i}}=\frac{\mathrm{X}_{\mathrm{i}}-\mathrm{X}_{\mathrm{i} 0}}{\delta \mathrm{X}_{\mathrm{i}}}
$$

où $x_{i}$ et $X_{i}$ représentent respectivement les valeurs codées et les valeurs naturelles des $\mathrm{i}^{\mathrm{e}}$ variables indépendantes, $\mathrm{X}_{\mathrm{i} 0}$ la valeur naturelle de la $\mathrm{i}^{\mathrm{e}}$ variable indépendante au point central et $\delta \mathrm{X}_{\mathrm{i}}$ la valeur du pas de variation correspondant.

Dans le but d'optimiser les paramètres photocatalytiques de réaction, chaque paramètre dans la conception (RSM) a été étudié à cinq niveaux différents $(-\alpha,-1,0,+1,+\alpha)$. Le choix de cinq niveaux pour chaque variable est exigé par cette conception afin d'explorer la région de la surface de réponse à proximité de l'optimum. Le plan central composite (CCD) (BOX et WILSON, 1951; BOX et HUNTER, 1987) est la conception expérimentale la plus employée pour modéliser une surface de réponse de second ordre.

Un plan CCD se compose de $\mathrm{N}_{\mathrm{f}}=2^{\mathrm{k}}$ points cubiques (plan factoriel ou fractionnaire), $2 \mathrm{k}$ points axiaux $( \pm \alpha, 0,0, \ldots, 0)$, $(0, \pm \alpha, 0, \ldots, 0),(0,0, \pm \alpha, \ldots, 0), \ldots,(0,0, \ldots, \pm \alpha)$ et un nombre de réplications choisi au centre du domaine $(0,0,0, \ldots, 0)$ ou points centraux, avec $\mathrm{k}$ le nombre de variables indépendantes (facteurs). Un CCD peut être rendu rotatif en choisissant la valeur appropriée de $\alpha$ soit $\alpha=\left(\mathrm{N}_{\mathrm{f}}\right)^{1 / 4}$.

Une conception expérimentale rotative signifie que la variance de la réponse prédite à un point $\mathrm{x}$ est fonction seulement de la distance du point au centre de la conception et ne dépend pas de la direction.

Pour nos essais, trois variables indépendantes sont étudiées. Par conséquent, pour $\mathrm{k}=3$, il faudrait 8 points cubiques, 6 points axiaux et 6 points centraux. Ce qui fait un total de 20 expériences nécessaires pour un modèle polynomial de second ordre. En utilisant trois facteurs, la valeur de $\alpha$ est égale à 1,68 .

Les paramètres choisis, dans le cadre de cette étude sont (en codé et naturel) : la concentration initiale en 4-méthylphénol $\left(\mathrm{x}_{1}, \mathrm{X}_{1}\right)$, la charge en catalyseur $\left(\mathrm{x}_{2}, \mathrm{X}_{2}\right)$ et le débit de circulation $\left(\mathrm{x}_{3}, \mathrm{X}_{3}\right)$. Les gammes et les niveaux de ces paramètres sont représentés dans le tableau 1. L'efficacité de la photodégradation constitue la variable de réponse.

\section{RÉSULTATS ET DISCUSSION}

La dégradation par photocatalyse est dépendante de plusieurs paramètres opératoires. Il peut être utile de connaître leur effet et de pouvoir le modéliser. L'utilisation de la méthodologie des plans d'expérience permettra de déterminer l'influence de plusieurs paramètres opératoires sur les performances de la photodégradation.

\subsection{Résultats de la conception expérimentale centrale composite}

Les résultats des expériences du plan central composite (CCD) sont représentés dans le tableau 2 qui montre les combinaisons statistiques des variables indépendantes $\mathrm{x}_{1}$ (concentration initiale en 4-méthylphénol), $\mathrm{x}_{2}$ (concentration en catalyseur), $\mathrm{x}_{3}$ (débit de circulation), avec les rendements (\%) observés (mesurés) et prédits sur une durée de 120 minutes. 
Tableau 1. Gammes et niveaux expérimentaux des variables indépendantes d'essais.

Table 1. Range and levels of experimental parameters.

\begin{tabular}{cccccc}
\hline Variables indépendantes & \multicolumn{5}{c}{ Gammes et niveaux } \\
\cline { 2 - 6 } & $\alpha$ & -1 & $\mathbf{0}$ & +1 & $+\alpha$ \\
\hline Concentration 4-méthylphénol $\left(\mu \mathrm{L}^{-1} \mathrm{~L}^{-1}\right)$ & 30 & 54,28 & 90 & 125,71 & 150 \\
Charge en catalyseur $\mathrm{ZnO}\left(\mathrm{g} \cdot \mathrm{L}^{-1}\right)$ & 0,5 & 1,01 & 1,75 & 2,49 & 3 \\
Débit de recirculation $\left(\mathrm{mL} \cdot \mathrm{s}^{-1}\right)$ & 0,5 & 1,21 & 2,26 & 3,31 & 4,03 \\
\hline
\end{tabular}

Tableau 2. Résultats du plan central composite (CCD) du rendement de la photodégradation du para-crésol et de la DCO pour trois variables d'essais indépendantes dans les unités codées et naturelles avec les réponses observées et prédites.

Table 2. CCD design matrix for three test variables in coded and natural units along with the observed and predicted responses

\begin{tabular}{|c|c|c|c|c|c|c|c|c|c|c|}
\hline \multirow[b]{2}{*}{ No exp. } & \multicolumn{3}{|c|}{ Valeurs codées } & \multicolumn{3}{|c|}{ Valeurs naturelles } & \multicolumn{4}{|c|}{ Rendement (\%) } \\
\hline & $\mathrm{x}_{1}$ & $\mathrm{x}_{2}$ & $\mathrm{x}_{3}$ & $\mathrm{X}_{1}$ & $\mathrm{X}_{2}$ & $\mathrm{X}_{3}$ & $\begin{array}{l}\text { Y(4-méthyl } \\
\text { phénol) }\end{array}$ & $\begin{array}{c}\mathrm{Y}_{\text {pred }}(4 \mathrm{méthyl} \\
\text { phénol })\end{array}$ & Y (DCO) & $\mathrm{Y}_{\mathrm{pred}}(\mathrm{DCO})$ \\
\hline 1 & -1 & -1 & -1 & 54,28 & 1,006 & 1,215 & 85,05 & 84,20 & 69,99 & 70,72 \\
\hline 2 & 1 & -1 & -1 & 125,71 & 1,006 & 1,215 & 69,75 & 69,35 & 63,10 & 61,59 \\
\hline 3 & -1 & 1 & -1 & 54,28 & 2,49 & 1,215 & 98,50 & 99,36 & 95,60 & 95,23 \\
\hline 4 & 1 & 1 & -1 & 125,71 & 2,49 & 1,215 & 77,39 & 76,70 & 65,60 & 65,46 \\
\hline 5 & -1 & -1 & 1 & 54,28 & 1,006 & 3,315 & 69,85 & 70,05 & 58,33 & 57,39 \\
\hline 6 & 1 & -1 & 1 & 125,71 & 1,006 & 3,315 & 52,25 & 50,91 & 48,25 & 47,54 \\
\hline 7 & -1 & 1 & 1 & 54,28 & 2,49 & 3,315 & 91,04 & 90,96 & 81,20 & 81,62 \\
\hline 8 & 1 & 1 & 1 & 125,71 & 2,49 & 3,315 & 63,65 & 64,01 & 52,94 & 51,13 \\
\hline 9 & $-\alpha$ & 0 & 0 & 30 & 1,75 & 2,26 & 96,30 & 95,99 & 85,00 & 84,58 \\
\hline 10 & $+\alpha$ & 0 & 0 & 150 & 1,75 & 2,26 & 59,85 & 60,85 & 49,30 & 51,26 \\
\hline 11 & $\begin{array}{c}+\alpha \\
0\end{array}$ & $-\alpha$ & 0 & 90 & 0,5 & 2,26 & 61,03 & 62,22 & 51,07 & 52,00 \\
\hline 12 & 0 & $+\alpha$ & 0 & 90 & 3 & 2,26 & 86,50 & 85,99 & 75,03 & 75,64 \\
\hline 13 & 0 & 0 & $-\alpha$ & 90 & 1,75 & 0,5 & 84,12 & 84,53 & 77,30 & 77,55 \\
\hline 14 & 0 & 0 & $+\alpha$ & 90 & 1,75 & 4,03 & 61,68 & 68,11 & 53,00 & 54,29 \\
\hline 15 & 0 & 0 & 0 & 90 & 1,75 & 2,26 & 68,52 & 68,11 & 58,95 & 58,47 \\
\hline 16 & 0 & 0 & 0 & 90 & 1,75 & 2,26 & 68,01 & 68,11 & 58,04 & 58,47 \\
\hline 17 & 0 & 0 & 0 & 90 & 1,75 & 2,26 & 68,45 & 68,11 & 59,45 & 58,47 \\
\hline 18 & 0 & 0 & 0 & 90 & 1,75 & 2,26 & 68,65 & 68,11 & 59,05 & 58,47 \\
\hline 19 & 0 & 0 & 0 & 90 & 1,75 & 2,26 & 67,10 & 68,11 & 57,65 & 58,47 \\
\hline 20 & 0 & 0 & 0 & 90 & 1,75 & 2,26 & 68,03 & 68,11 & 57,97 & 58,47 \\
\hline
\end{tabular}

Les résultats de la conception expérimentale ont été étudiés et interprétés par le logiciel MINITAB 14 (PA, USA) pour estimer la réponse de la variable dépendante dans l'ensemble des expériences.

La conception centrale composite (CCD) se compose de trois variables indépendantes citées plus haut. Les réponses sont l'abattement (\%) du 4-méthylphénol et de la DCO. Elles sont représentées par les modèles de second ordre suivants :

$$
\begin{aligned}
& Y_{\text {pred }}(4-\text { méthylphénol })=68,107+(-10,449) \mathrm{x}_{1} \\
& +(7,067) \mathrm{x}_{2}+(-6,710) \mathrm{x}_{3}+(-1,95) \mathrm{x}_{1} \mathrm{x}_{2} \\
& +(-1,073) \mathrm{x}_{1} \mathrm{x}_{3}+(1,438) \mathrm{x}_{2} \mathrm{x}_{3}+(3,646) \mathrm{x}_{1}{ }^{2} \\
& +(2,122) \mathrm{x}_{2}{ }^{2}+(1,816) \mathrm{x}_{3}{ }^{2}
\end{aligned}
$$

$$
\begin{aligned}
& Y_{\text {pred }}(D C O)=58,4744+(-9,9049) \mathrm{x}_{1} \\
& +(7,0269) \mathrm{x}_{2}+(-6,9150) \mathrm{x}_{3} \\
& +(-5,1613) \mathrm{x}_{1} \mathrm{x}_{2}+(-0,1813) \mathrm{x}_{1} \mathrm{x}_{3} \\
& +(-0,0688) \mathrm{x}_{2} \mathrm{x}_{3}+(3,3386) \mathrm{x}_{1}{ }^{2} \\
& +(1,8890) \mathrm{x}_{2}{ }^{2}+(2,6315) \mathrm{x}_{3}{ }^{2}
\end{aligned}
$$

En traçant les courbes de parité donnant les valeurs prédites en fonction des valeurs observées (Figures $2 a$ et $2 b$ ), on constate que le modèle polynomial d'ordre deux corrèle bien les résultats expérimentaux.

L'analyse de la variance (ANOVA) du modèle de régression quadratique montre que les modèles sont hautement significatifs au seuil de $1 \%$. Ceci est justifié par le fait que la 


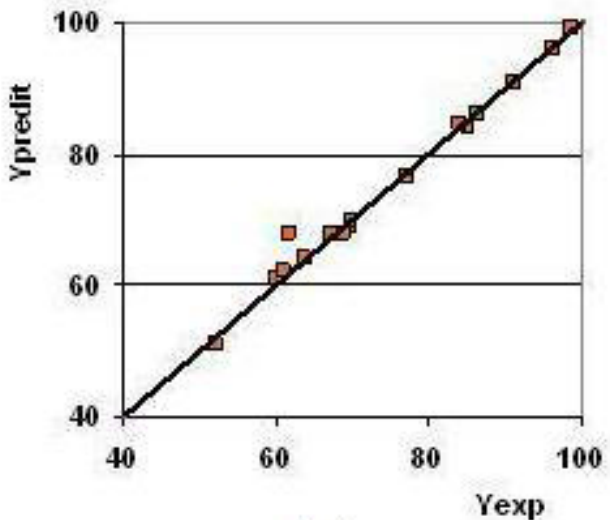

(a)

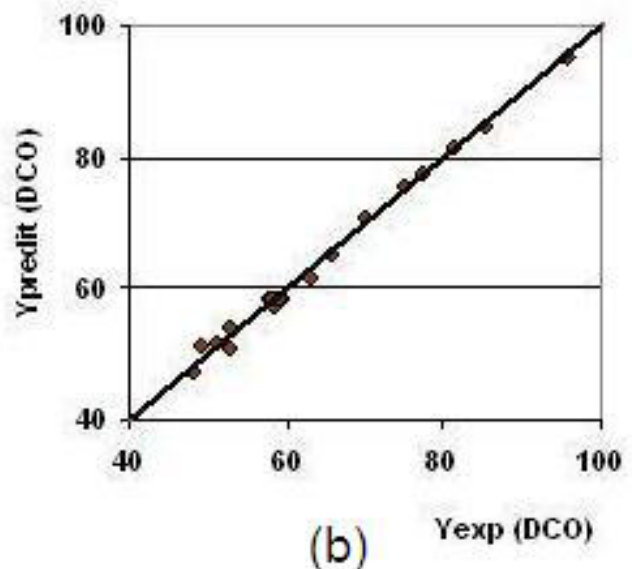

(b)

Figure 2. Comparaison des valeurs prédites et observées pour les rendements (a) en 4 méthylphénol et (b) en DCO. Comparison of the observed values and the predicted values for elimination of (a) 4 methylphenol and (b) COD.

valeur du F calculée est beaucoup plus grande que la valeur de F tabulée soit :

$$
\begin{aligned}
\mathrm{F}_{0,01 ; 9 ; 10}(4-\text { méthylphénol }) & =\mathrm{S}^{2}{ }_{\text {reg }} / \mathrm{S}^{2}{ }_{\text {err }}= \\
396,28 \gg \mathrm{F}_{0,01 ; 9 ; 10(\text { tabulée })} & =4,94 \\
\mathrm{~F}_{0,01 ; 9 ; 10}(\mathrm{DCO}) & =200,54 \gg \\
\mathrm{F}_{0,01 ; 9 ; 10(\text { tabulée })} & =4,94
\end{aligned}
$$

Les probabilités (P-valeurs) indiquent la signification du modèle (Tableau 3). La P-valeur relative au modèle de régression est très faible ( $\mathrm{P}$-valeur $=0,000)$, ce qui montre la signification du modèle. De plus, la contribution de chacun des termes linéaires, quadratiques et interactions est hautement significative au seuil de $1 \%$ (P-valeur < 0,01). Par conséquent, on peut conclure que les deux modèles de prédiction (Eq. 5 et 6) sont hautement significatifs et estiment de manière adéquate les données observées.

Les valeurs des coefficients de détermination respectivement du 4-méthylphénol et de la DCO sont $\mathrm{R}^{2}=0,997$ et $\mathrm{R}^{2}=0,994$. Ces valeurs élevées indiquent que $99,7 \%$ et $99,4 \%$ d'une simple variation du rendement photocatalytique du 4-méthylphénol et de la DCO sont expliquées par les variables indépendantes. Les valeurs du coefficient de détermination ajustées sont également très élevées, soit $\mathrm{R}_{\text {adj }}^{2}$ (4-méthylphénol) = 99,5\% et $\mathrm{R}_{\text {adj }}(\mathrm{DCO})=99 \%$, suggérant une haute signification des modèles (Eq. 5 et 6 ) et un bon ajustement de ces derniers aux données expérimentales.

\subsection{Graphes des effets des facteurs}

Les effets individuels, quadratiques et interactions des différents facteurs ont été estimés (Tableau 4). Un coefficient avec un signe (+) signifie que le facteur a un effet synergétique. Par contre, un signe (-) met en évidence un effet antagoniste du facteur.

L'effet individuel de la concentration en catalyseur est positif sur le rendement photocatalytique du fait que le coefficient correspondant est positif. À noter, nous n'avons pas observé d'effet de photocorrosion du $\mathrm{ZnO}$ lors de nos différents essais.

Par contre, la concentration initiale en 4-méthylphénol a un effet négatif important sur la photodégradation car le

Tableau 3. Analyse (ANOVA) pour le rendement de photodégradation du 4-méthylphénol ( $\%$ rendement) et pour l'abattement de la DCO (\% DCO).

Table 3. Analysis of variance (ANOVA) for 4-methyl phenol degradation (\%) and COD elimination (\%COD).

\begin{tabular}{cccccc}
\hline Source & $\begin{array}{c}\text { Degrés de } \\
\text { liberté }\end{array}$ & $\begin{array}{c}\text { F-valeur } \\
\text { (\% rendement) }\end{array}$ & $\begin{array}{c}\text { P-valeur } \\
\text { (\% rendement) }\end{array}$ & $\begin{array}{c}\text { F-valeur } \\
\text { (\% DCO) }\end{array}$ & $\begin{array}{c}\text { P-valeur } \\
\text { (\% DCO) }\end{array}$ \\
\hline Régression (modèle) & 9 & 396,28 & 0,000 & 200,54 & 0,000 \\
Linéaire & 3 & 1067,32 & 0,000 & 510,23 & 0,000 \\
Quadratique & 3 & 100,02 & 0,000 & 50,57 & 0,000 \\
Interactions & 3 & 21,50 & 0,000 & 40,82 & 0,000 \\
\hline
\end{tabular}


Tableau 4. Estimation des coefficients de régression pour le rendement d'élimination du 4-méthylphénol $\left(\mathrm{Y}_{1}\right)$ et pour l'abattement en DCO $\left(\mathrm{Y}_{2}\right)$ en utilisant les valeurs codées.

Table 4. Factor effects and associated p-values for two responses.

\begin{tabular}{|c|c|c|c|c|c|c|}
\hline \multirow{3}{*}{ Relations } & & \multirow{3}{*}{ Facteur } & \multicolumn{4}{|c|}{ Réponses } \\
\hline & & & \multicolumn{2}{|c|}{$Y_{1}$} & \multicolumn{2}{|c|}{$Y_{2}$} \\
\hline & & & Effet facteur & p-valeur & Effet facteur & p-valeur \\
\hline \multirow{3}{*}{$\begin{array}{c}\text { Effets } \\
\text { principaux }\end{array}$} & Linéaire & $\mathrm{X}_{1}$ & $-41,382$ & 0,000 & $-27,729$ & 0,000 \\
\hline & Linéaire & $\mathrm{X}_{2}$ & 27,988 & 0,000 & 19,672 & 0,000 \\
\hline & Linéaire & $x_{3}^{2}$ & $-26,574$ & 0,000 & $-19,672$ & 0,000 \\
\hline \multirow{6}{*}{ Interactions } & & $\mathrm{X}_{1}^{2}$ & 14,833 & 0,000 & 9,601 & 0,000 \\
\hline & Quadratique & $\mathrm{X}_{2}^{2}$ & 8,633 & 0,000 & 5,433 & 0,000 \\
\hline & & $\mathrm{X}_{3}^{2}$ & 7,389 & 0,000 & 7,568 & 0,000 \\
\hline & & $\mathrm{X}_{1} \mathrm{xx}_{2}$ & $-5,911$ & 0,000 & $-22,059$ & 0,000 \\
\hline & Croisées & $\mathrm{x}_{1}^{1} \mathrm{xx}_{3}^{2}$ & $-3,251$ & 0,009 & 0,388 & 0,706 \\
\hline & & $\mathrm{X}_{2} \mathrm{xx}_{3}$ & 4,357 & 0,001 & $-0,147$ & 0,866 \\
\hline
\end{tabular}

coefficient correspondant est affecté du signe négatif. La gamme de concentrations étudiée varie de 30 à $150 \mu \mathrm{L} \mathrm{L}^{-1}$ (Tableau 1 ). Ceci paraît évident car plus on augmente la concentration en polluant, plus le rendement de photodégradation décroît en raison de la quantité de produit à traiter. Le débit de recirculation influe aussi négativement sur les performances $\mathrm{du}$ procédé. Ceci est dû probablement à la diminution du temps de séjour dans le réacteur.

Sur les graphes des effets (Figure 3), on visualise l'évolution $\mathrm{du}$ rendement photocatalytique en fonction de chacun des paramètres opératoires étudiés.

On note bien l'effet antagoniste de la concentration initiale en polluant $\left(\mathrm{x}_{1}\right)$ et du débit de recirculation $\left(\mathrm{x}_{3}\right)$. La concentration en catalyseur $\left(\mathrm{x}_{2}\right)$ a un effet positif dans l'intervalle étudié. On signalera que le même type de comportement est observé concernant l'abattement en DCO.

\subsection{Optimisation des paramètres}

L'étude des graphes de contours fournit une méthode simple d'optimisation du taux de traitement et d'identification des interactions entre les variables (voir Figures 4 et 5). Chaque courbe représente, dans notre cas, une infinité de combinaisons entre deux variables quand la troisième variable est maintenue à un niveau constant. Ces niveaux constants sont les niveaux centraux de chacune des variables.

Les principales informations que l'on peut en tirer concernant les conditions optimales de fonctionnement du procédé sont :
- L'efficacité du rendement de photodégradation est inversement affectée par la concentration initiale en 4-méthylphénol (Figure 4a). Ce qui est assez couramment observé en photocatalyse, l'augmentation de la cinétique de dégradation ne compense pas l'augmentation de la quantité de produit à dégrader.

- L'augmentation de la concentration en $\mathrm{ZnO}$ entraîne une augmentation du rendement (Figure $4 \mathrm{~b}$ ). L'effet de la quantité de catalyseur est toujours positif dans la gamme de concentration choisie.

- La diminution du débit de circulation entraîne l'accroissement du rendement de dégradation (Figure 4c). Ceci s'explique par l'augmentation du temps de séjour de la solution dans le réacteur.

- L'effet le plus important est celui de la concentration initiale en 4-méthylphénol, suivie par la concentration en catalyseur et enfin par le débit de recirculation.

Les effets linéaires, quadratiques et les interactions sont significatifs pour les deux réponses, hormis les interactions $\left(\mathrm{x}_{1} \mathrm{x}_{3}\right)$ et $\left(\mathrm{x}_{2} \mathrm{x}_{3}\right)$ pour la $\mathrm{DCO}$, où on remarque un faible effet (Figures $5 \mathrm{~b}$ et $5 \mathrm{c}$ ). Avec une concentration élevée en catalyseur et un faible débit de circulation de la solution, fixée à la moyenne dans l'intervalle du domaine expérimental choisi, une dégradation complète est atteinte pour une faible concentration initiale en 4-méthylphénol. Les valeurs optimales conduisant à un rendement de $100 \%$ pour les deux réponses sont vérifiées dans notre cas et elles apparaissent sur les figures $4 \mathrm{a}$ et $5 \mathrm{a}$. Les valeurs naturelles correspondantes sont respectivement : [4-méthylphénol] $=\left(48,16 \mu \mathrm{L}^{\circ} \mathrm{L}^{-1}\right.$; $\left.43,76 \mu \mathrm{L} \cdot \mathrm{L}^{-1}\right) ;[\mathrm{ZnO}]=\left(2,34 \mathrm{mg} \bullet \mathrm{L}^{-1} ; 2,42 \mathrm{mg} \bullet \mathrm{L}^{-1}\right)$ et débit de circulation $=\left(1,24 \mathrm{~mL} \cdot \mathrm{s}^{-1} ; 1,22 \mathrm{~mL} \bullet \mathrm{s}^{-1}\right)$. Une validation expérimentale de ces conditions optimales a été réalisée. 


\section{Effets principaux des facteurs pour le rendement de dégradation}

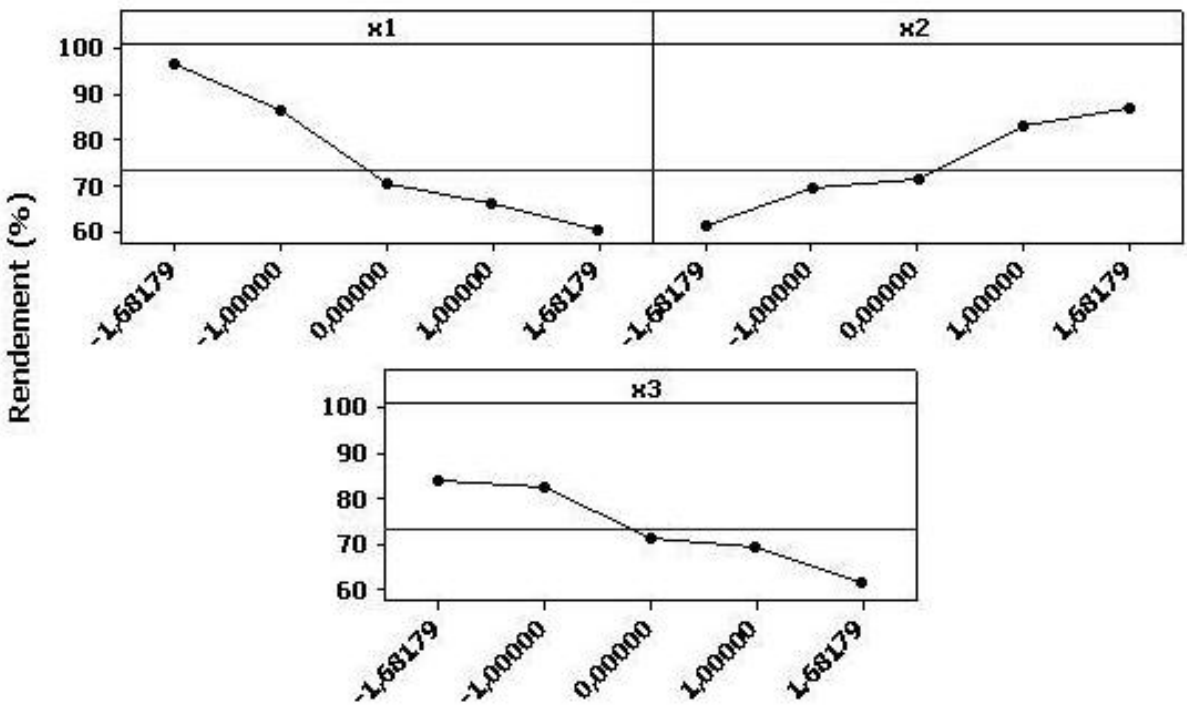

Figure 3. Graphes des effets des facteurs sur le rendement de photodégradation à $t=120$ min $\left(x_{1}=\right.$ concentration en 4-méthylphénol, $x_{2}=$ concentration en $\mathrm{ZnO}, x_{3}=$ débit de recirculation).

Two-way interaction plots for 4 -methylphenol removal at $t=120$ min $\left(x_{1}=\right.$ concentration of 4-méthylphénol, $x_{2}=$ concentration of $\mathrm{ZnO}, x_{3}=$ recirculating flow).

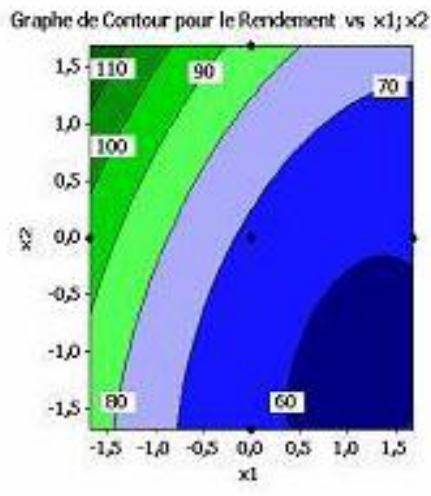

(a)

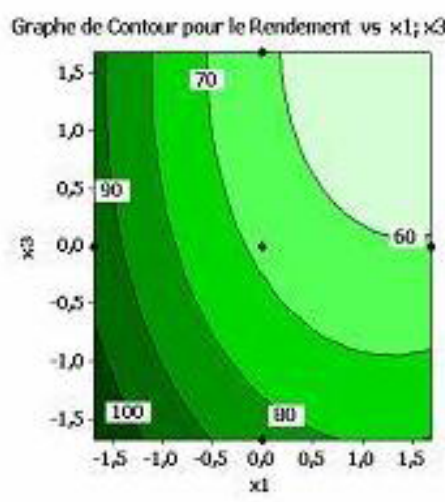

(b)

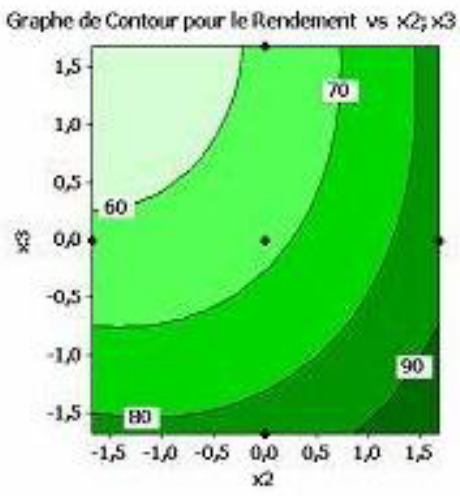

(c)

Figure 4. Graphes de contours pour le rendement de photodégradation de 4 méthylphénol (\%) pour chaque paire de variables en valeurs codées lorsque la troisième variable est fixée à la moyenne.

Contour plots of 4-methylphenol removal (\%) for each pair of variables in coded values when the third variable is held constant at the mean value.

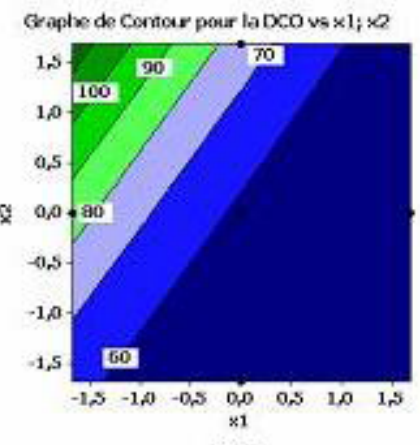

(a)

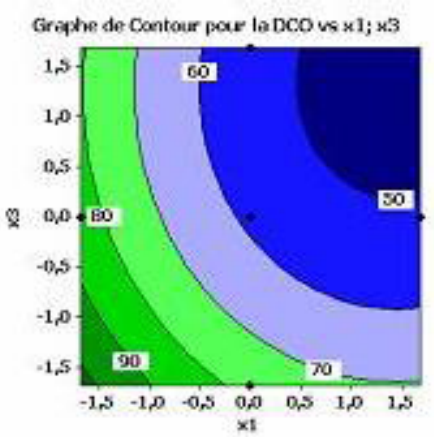

(b)

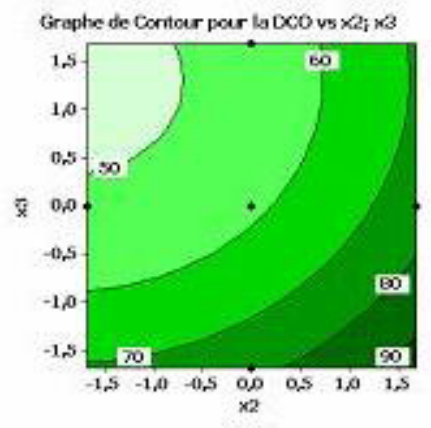

(c)

Figure 5. Graphes de contours du rendement de la demande chimique en oxygène DCO (\%) pour chaque paire de variables en valeurs codées lorsque la troisième variable est fixée à la moyenne.

Contour plots of COD removal (\%) for each pair of variables in coded values when the third variable is held constant at the mean value. 


\section{CONCLUSION}

La dégradation photocatalytique du 4-méthylphénol en solution aqueuse est étudiée dans un réacteur en circulation. L'influence des trois paramètres, la concentration initiale en polluant, la charge en catalyseur et le débit de circulation, furent étudiés. Les résultats montrent que l'application de la méthodologie des surfaces de réponses (RSM) et le plan central composite (CCD) permettent de décrire et de modéliser d'une manière correcte l'influence de ces trois paramètres expérimentaux sur l'efficacité du traitement. Les valeurs optimales des paramètres donnant un rendement maximal (100\%) ont ainsi pu être déterminées. La fiabilité des deux modèles de prévision de second ordre fondée sur la régression multiple a été testée par la méthode d'analyse (ANOVA). Cette analyse a montré que les modèles sont hautement significatifs et en bonne adéquation avec les résultats expérimentaux.

\section{RÉFÉRENCES BIBLIOGRAPHIQUES}

AMERICAN PUBLIC HEALTH ASSOCIATION (1998). Standard methods for the examination of water and wastewater, $20^{\text {th }}$ ed., USA.

BOX G.E.P. et W.G. HUNTER (1987). Statistics for experimenters: an introduction to design, data analysis, and model building, Wiley, New York, USA, ?? p.

BOX G.E.P. et K.B. WILSON (1951). On the experimental attainment of optimum conditions, J. R. Stat. Soc., Ser. B Stat. Meth., 13, 1-45.

CHO I.H. et K.D. ZOH (2007). Photocatalytic degradation of azo dye (reactive red 120) in $\mathrm{TiO}_{2} / \mathrm{UV}$ system: Optimization and modelling using a response surface methodology (RSM) based on central composite design, Dyes Pigm.,75, 533-543.

HERRERA F., A. LOPEZ et J. KIWI (2000). Photochemically activated degradation of reactive dyes. Statistical modelling of the reactor performance, J. Photochem. Photobiol. A: Chemistry, 135, 45-51.

KHODJA A.A., T. SEHILI, J.F. PITICHOWSKI et P. BOULE (2001). Photocatalytic degradation of 2-phenylphenol on $\mathrm{TiO}_{2}$ and $\mathrm{ZnO}$ in aqueous suspensions, J. Photochem. Photobiol. A: Chemistry, 141, 231-239.

KHURI A.I. et J.A. CORNEL (1987). Response Surfaces: Design and Analysis, MARCEL DEKKER (Éditeur), New York, NY, USA.
KUMAR S.A. et S. KANMANI (2006). Studies on solar photocatalytic degradation of phenol using response surface methodology. Dans : $1^{\text {st }}$ European Conference on EAAOP, Chania, Grèce.

MARCI G., V. AUGUGLIARO, M.J.L. MUNOZ, C. MARTIN, L. PALMISANO, V. RIVES, M. SCHIAVELLO et R.J.D. TILLEY (2001). Preparation characterization and photocatalytic activity of polycrystalline $\mathrm{ZnO} / \mathrm{TiO}_{2}$ systems. 1. Surface and bulk characterization, J. Phys. Chem., 105, 1026-1032.

MONTGOMERY D.C. (2001). Design and analysis of experiments. WILEY \& SONS (Éditeurs), New York, NT, USA, ?? p.

MYERS R.H. et D.C. MONTGOMERY (2002). Response Surface Methodology, $2^{\text {nd }}$ Ed., WILEY C SONS (Éditeurs), New York, NY, USA, ?? p.

OLIVEROS E., O. LEGRINI, M. HOHL, T. MULLER et A.M. BRAUN (1997). Wat. Sci. Technol., 35, 223.

OLLIS D.F. et H. AL-EKABI (1993). Photocatalysis purification and treatment of water and air. ELSEVIER (Éditeur), Amsterdam, NL, ?? p.

PEREZ M., F. TORRADES, J. PERAL, C. LIZAMA, C. BRAVO, S. CASAS, J. FREER et H.D. MANSILLA (2001). Multivariate approach to photocatalytic degradation of a cellulose bleaching effluent, Appl. Cat. B: Environ., 33, 89-96.

PIRKANNIEMI K. et SILLANPAA (2002). Heterogeneous water phase catalysis as an environmental application: a review. Chemosphere, 48, 1047-1060.

SCHIAVELLO M. (1997). Heterogeneous photocatalysis. WILEY \& SONS (Éditeurs), New York, NT, USA, ?? p.

YASSITEPE E., H.C. YATMAZ, C. OZTURK, K. OZTURK et C. DURAN (2008). Photocatalytic efficiency of $\mathrm{ZnO}$ plates in degradation of azo dye solutions, J. Photochem. Photobiol. A: Chem., 198, 1-6. 